\title{
Intelligent Presentation Skills Trainer Analyses Body Movement
}

\author{
Anh-Tuan Nguyen ${ }^{(凶)}$, Wei Chen, and Matthias Rauterberg \\ Department of Industrial Design, Eindhoven University of Technology, \\ Postbus 513, 5600MB Eindhoven, The Netherlands \\ \{a.nguyen, w. chen, g.w.m.rauterberg\}@tue.nl
}

\begin{abstract}
Public speaking is a non-trivial task since it is affected by how nonverbal behaviors are expressed. Practicing to deliver the appropriate expressions is difficult while they are mostly given subconsciously. This paper presents our empirical study on the nonverbal behaviors of presenters. Such information was used as the ground truth to develop an intelligent tutoring system. The system can capture bodily characteristics of presenters via a depth camera, interpret this information in order to assess the quality of the presentation, and then give feedbacks to users. Feedbacks are delivered immediately through a virtual conference room, in which the reactions of the simulated avatars can be controlled based on the performance of presenters.
\end{abstract}

Keywords: Body motion analysis $\cdot$ Depth vision $\cdot$ Nonverbal behavior • Social signal processing

\section{Introduction}

Public speaking is the art of persuasion. It has the tremendous impact on the success of everyone [1, p.102]. Unfortunately, delivering an oral presentation is not as simple as computer data transmission. Instead, the audience simultaneously perceives the messages via various non-spoken channels, which are known as nonverbal behaviors. On one hand, the content of a presentation must be clear, vivid and appropriate [2]. On the other hand, the significant component of a presentation lies upon nonverbal cues, which has the power to change the meaning assigned to the spoken words [1, p.241].

Nonverbal behaviors of public speakers are expressed via several channels such as voice, gesture and facial expression. They have been proven to have greater influence than verbal cues. For example, a research by [3] showed that, nonverbal messages are twelve to thirteen times more powerful than verbal ones. Similarly, according to [4], the audience receives more than half of information from body language. The same result was found during the study of [1], in which most people unconsciously more believe in nonverbal than verbal communication.

Practicing to express the effective nonverbal behaviors is difficult due to the fact that, they are mostly expressed subconsciously. Thus, in order to achieve the

(C) Springer International Publishing Switzerland 2015

I. Rojas et al. (Eds.): IWANN 2015, Part II, LNCS 9095, pp. 320-332, 2015.

DOI: $10.1007 / 978-3-319-19222-2 \_27$ 
positive learning results, learners must be provided with the appropriate feedbacks from skilled experts, which in most cases might be expensive to achieve. In parallel, the role of nonverbal behaviors in computing is becoming increasingly recognized by the development of the emerging fields, such as social signal processing [5] and affective computing [6]. Therefore, computers have been equipped with the abilities to decode the complexity of humans non-spoken channels.

In the literature, there are several approaches toward the automatic recognition of nonverbal cues from presenters, such as [7-10]. These approaches analyze some vocal and visual channels of presenters, thus can provide them with the information about their performance. For example, the system in [10] was built solely on vocal cues, by analyzing the physical characteristics of voice such as pitch or tempo. It was similar to the approach of [9], which was originated from a vocal emotion detection module. The authors applied the support vector machine [11] to analyze one presentation based on a set of 6 qualities and achieved the accuracy of $81 \%$. The approach introduced in [7] might be the simplest. By relying on the importance of pitch variance in oral presentations, the system measured the changes in vocal pitch, and then give visual feedbacks to promote pitch variation.

On the other hand, there are three systems that include visual cues in the analysis. In [8], the authors added face position and orientation as the approximation of eye contact, together with utterance, pitch, filled pauses and speaking rate. In contrast, [12] introduced the method that only based on visual information. Similar to [8], face orientation was used as an indication for eye contact. The authors tracked the trajectories of global body movement and head position. This information helped their system to rank the performance of the whole presentation using the RankBoost algorithm [13], achieved promising results. However, they did not consider the complexity of body parts. To the best of our knowledge, the system that was presented in [14] is the only one that included the configurations of single body parts.

The common drawback of most existing systems is that, they were not implemented based on empirical research of nonverbal behaviors. Moreover, although most of them provided mechanisms to deliver feedbacks to the presenters (except [9]), the forms of feedbacks are rather simple. They are text/images [8], sound [10] or lightning [7]. These methods can only provide users with solely assessment information, without concerning the entertaining aspect of the system, which might be valuable for educational purposes.

This paper presents our progress in developing a tutoring system for public speaking, which assesses presentations based solely on the visual behaviors of presenters. Firstly, an empirical study was performed in order to investigate on the nonverbal cues that impact a presentation, serving as the ground truth. Next, a Microsoft Kinect was implemented for capturing skeletal representations of the presenters' body as input data for the analysis. The recognition process can detect if the behaviors appeared in real-time. Multi-class support vector machine was used to classify the quality of presentations into a four-degree scale with the recognition rate of $73.9 \%$ on a training/test database that includes 
76 presentations. For the feedback, the system allows presenters to review their presentation, together with the analysis results. In parallel, we developed a simulated conference room as the real-time feedback mechanism.

In the next section, we will explain our empirical results from the recorded presentations. The current development status will be introduced afterward. The last section is for conclusions and future works.

\section{Nonverbal Behaviour of Presenters}

In order to gather the ground truth as the guidance for our system, an observation was performed. We collected data from a training class about public speaking skills for postgraduate students. Learners were asked to give short presentations (about one minute) in front of the audience, which includes about ten other learners and one or two coaches. The content of the presentations was freely chosen by the presenters. In fact, all presenters chose to talk about their own research, in the ways that it can be understood by all of the audience that might came from the different fields. After each presentation, the audience gave feedbacks and suggestions on how the presentation should be improved, in terms of nonverbal expressions. We set up a regular camera to record the presentations. In parallel, a Microsoft Kinect was used to capture the wholebody movement for our further signal processing, as well as behavioral studies (Figure 1). Data from Kinect was stored as the *.ONI files using the OpenNI SDK (http://www.openni.org/). Finally, after removed the unsatisfied videos (e.g. presenters moved out of the camera range), 39 presentations of 11 presenters (four females, seven males) were collected.

Regular videos were used for behavioral analysis. This task was done through the collaboration with an expert in public speaking. The role of the expert was to review the recorded videos, and then specifying the nonverbal cues that affected the performance of the speakers, together with the durations that they appeared. Thus, for each video, a set of behaviors was created. We collected the nonverbal cues and then annotated their appearance using the commercial software Noldus Observer XT [15]. Behaviors were categorized into either State event if their duration is necessary to be studied, or Point event otherwise. The software provided us with the statistical analysis on the appearance of these behaviors, including the number of presentations that contain the behaviors, the rate that they appeared (point events) and the percentage of time that they accounted for (Table 1).

The observed behaviors can be separated based on the nonverbal channels that they were generated: (1) Posture (the static configuration of body), (2) Voice (concerning the paralinguistic characteristics), (3) Eye contact, (4) Facial Expression, (5) Globe body movement, (6) Hand gesture. This method of categorization is similar to the literature of public speaking skills [2]. Due to the limited amount of space, we could not describe all of the observed behaviors in 
Table 1. The list of observed nonverbal cues

\begin{tabular}{|c|c|c|c|c|c|c|c|c|}
\hline \multirow[t]{2}{*}{ \# Behaviors } & \multirow{2}{*}{$\begin{array}{c}\text { Event } \\
\text { Type } \\
(\mathrm{S} / \mathrm{P})\end{array}$} & \multirow{2}{*}{ No. } & \multicolumn{3}{|c|}{$\begin{array}{c}\text { Rate of occurrences } \\
\text { (times/minute) }\end{array}$} & \multicolumn{3}{|c|}{$\begin{array}{l}\text { Percentage during observation } \\
\text { of the occurrences }(\%)\end{array}$} \\
\hline & & & $\mathrm{M}$ & $\mathrm{SD}$ & Range & $\mathrm{M}$ & SD & Range \\
\hline \multicolumn{9}{|l|}{ Postural behaviors } \\
\hline 1 (-) Shoulders too tight & $\mathrm{S}$ & 19 & & & & 60.94 & 23.80 & $12.67-98.50$ \\
\hline (-) Legs closed & $\mathrm{S}$ & 12 & & & & 73.02 & 36.44 & $5.15-100$ \\
\hline (-) Legs too stretch & $\mathrm{S}$ & 3 & & & & 61.42 & 11.33 & $19.18-100$ \\
\hline 4 (-) Weight in one foot & $\mathrm{S}$ & 20 & & & & 65.42 & 28.69 & $5.20-100$ \\
\hline 5 (-) Chin too high & $\mathrm{S}$ & 14 & & & & 64.94 & 23.80 & $12.67-98.50$ \\
\hline (-) Hands in pockets & $\mathrm{S}$ & 3 & & & & 11.85 & 4.89 & $12.76-96.20$ \\
\hline$(+)$ Lean forward & $\mathrm{S}$ & 19 & & & & 32.50 & 28.66 & $3.70-82.78$ \\
\hline 8 (-) Lean backward & $\mathrm{S}$ & 17 & & & & 62.80 & 28.07 & $12.73-96.20$ \\
\hline \multicolumn{9}{|l|}{ Vocal behaviors } \\
\hline 9 (-) Speak too fast & $\mathrm{S}$ & 19 & & & & 45.88 & 36.55 & $7.32-100$ \\
\hline $10(-)$ Start too fast & $\mathrm{P}$ & 18 & & & & & & \\
\hline $\begin{array}{l}11(-) \text { Energy decreases at the } \\
\text { end }\end{array}$ & $\mathrm{P}$ & 23 & 2.88 & 1.77 & $0.53-6.31$ & & & \\
\hline $12(+)$ Vocal emphasis & $\mathrm{P}$ & 33 & 5.51 & 4.51 & $0.59-17.50$ & & & \\
\hline $13(+)$ Suitable pause & $\mathrm{P}$ & 33 & 4.63 & 3.16 & $0.53-12.5$ & & & \\
\hline 14 (-) Unsuitable pause & $\mathrm{P}$ & 20 & 1.73 & 1.14 & $0.53-5.19$ & & & \\
\hline 15 (-) Monotone & $\mathrm{S}$ & 20 & & & & 92.49 & 13.08 & $56.29-100$ \\
\hline $16(-)$ Fillers & $\mathrm{P}$ & 34 & 5.17 & 4.22 & $1.44-19.03$ & & & \\
\hline $17(-)$ Stuttering & $\mathrm{P}$ & 12 & 1.72 & 0.83 & $0.53-3.42$ & & & \\
\hline \multicolumn{9}{|l|}{ Behaviors of eye contact } \\
\hline $18(+)$ Make eye contact & $\mathrm{S}$ & 39 & & & & 93.81 & 8.24 & $75.00-100$ \\
\hline 19 (-) Contact avoidance & $\mathrm{S}$ & 28 & & & & 9.98 & 8.47 & $1.12-25.00$ \\
\hline $19.1 \quad(-)$ Look up to ceiling & $\mathrm{S}$ & 14 & & & & 4.23 & 2.95 & $1.12-9.61$ \\
\hline 19.2 (-) Look down to floor & $\mathrm{S}$ & 19 & & & & 7.67 & 4.67 & $2.84-14.17$ \\
\hline $19.3 \quad(-)$ Look at hands & S & 11 & & & & 10.24 & 3.15 & $4.40-13.15$ \\
\hline \multicolumn{9}{|c|}{ Behaviors related to facial expression } \\
\hline $20(+)$ Facial mimicry & S & 30 & & & & 39.31 & 25.97 & $4.50-91.81$ \\
\hline $21(+)$ Smile & $\mathrm{S}$ & 22 & & & & 13.62 & 11.54 & $3.54-41.08$ \\
\hline $22(-)$ Flat face & $\mathrm{S}$ & 8 & & & & 80.61 & 24.16 & $40.41-100$ \\
\hline \multicolumn{9}{|c|}{ Behaviors related to whole body movement } \\
\hline 23 (-) Too much movement & $\mathrm{P}$ & 11 & & & & 42.21 & 25.87 & $4.68-89.32$ \\
\hline $24(-)$ Too little movement & $\mathrm{P}$ & 23 & & & & 50.62 & 29.21 & $10.05-100$ \\
\hline 25 (-) Step backward & $\mathrm{P}$ & 31 & 1.83 & 1.27 & $0.36-4.36$ & & & \\
\hline $26(+)$ Step forward & $\mathrm{P}$ & 34 & 2.06 & 1.04 & $0.59-4.61$ & & & \\
\hline \multicolumn{9}{|c|}{ Behaviors related to hand gesture } \\
\hline $\begin{array}{l}\text { Amount of hand gesture } \\
27 \text { Hand gesture occur }\end{array}$ & $\mathrm{P}$ & 38 & 16.83 & 7.15 & $0.93-28.42$ & & & \\
\hline 28 (-) Too little gestures & $\mathrm{S}$ & 20 & & & & 69.55 & 34.64 & $17.21-100$ \\
\hline 29 (-) Too much gestures & $\mathrm{S}$ & 10 & & & & 61.49 & 31.82 & $27.34-96.10$ \\
\hline \multicolumn{9}{|l|}{ Quality of hand gesture } \\
\hline 30 (-) Bounded gestures & $\mathrm{P}$ & 30 & 6.75 & 5.33 & $1.00-19.77$ & & & \\
\hline 31 (+) Relaxed gestures & $\mathrm{P}$ & 29 & 7.41 & 4.95 & $1.15-15.79$ & & & \\
\hline 32 (-) Casual gestures & $\mathrm{P}$ & 10 & 5.16 & 3.14 & $1.56-10.28$ & & & \\
\hline 33 (-) Uncompleted gestures & $\mathrm{P}$ & 27 & 3.23 & 2.78 & $0.93-10.27$ & & & \\
\hline $34(+)$ Gestural emphasis & $\mathrm{P}$ & 20 & 4.43 & 4.05 & $0.36-11.99$ & & & \\
\hline 35 (-) Repeated gestures & $\mathrm{P}$ & 31 & 6.57 & 2.49 & $1.09-12.31$ & & & \\
\hline
\end{tabular}

detail in this section. Only the behaviors that support our current development will be further explained in the next section. On the other hand, although we aimed to observe all of the available nonverbal cues, the contributions of each 

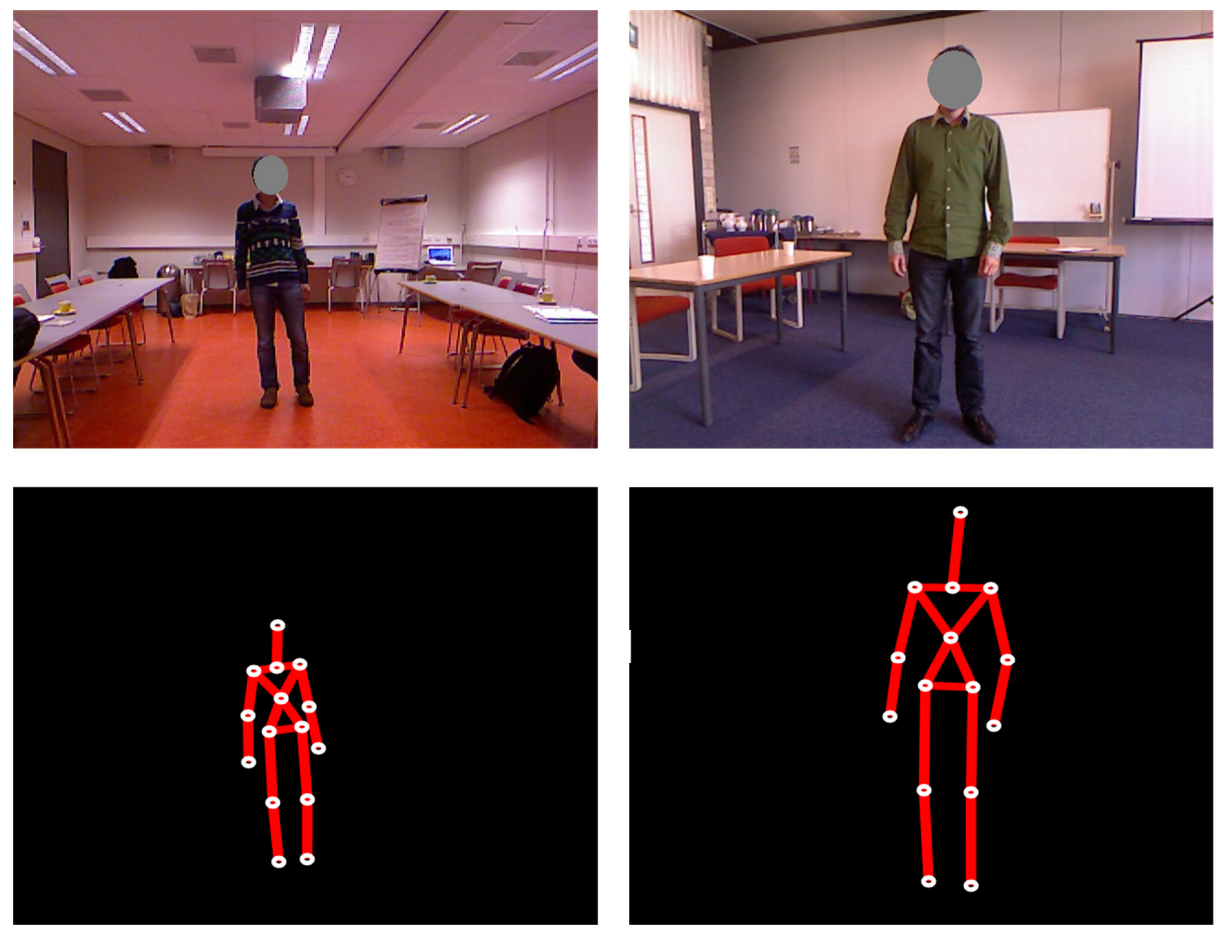

Fig. 1. Two samples from the database, with the color images (top row) and the skeletal representations of presenters' bodies, which were extracted and stored using Microsoft Kinect and the OpenNI SDK (bottom row)

individual to the success of a presentation are unequal. From our observation, as well as advices from the expert, the following aspects are the most important:

- Eye Contact: Similar to social interaction, maintaining good eye contact is the first thing the presenters must keep in mind. It initiates and strengthens the connection between them and the audience (\#18, 19 in Table 1). It might have the first and foremost influence to the performance of a presentation, as well as regular communications [16].

- Amount of energy: This aspect concerns the dynamic characteristics of a presentation, thus can reflect the internal state of the presenters. It has impact in most behaviors that we have found (except posture as the static channel). For example, the amount of whole body movement (\#23, 24), the amount of hand gesture (\#28, 29), vocal behaviors (partly via tempo, emphases) and most features of hand gesture.

- Variety: The presentations with strong variations significantly increase the attention of the audience. Lacking variation results in monotone (\#15), flat face (\#22), and hand gesture repeated (\#35). In fact, variety can be separated as one single measurement to analyze a presentation. It takes the role 
as rhythm in music. Even a beautiful piece of music, without changes in rhythm will steadily lose attention from the audience.

\section{Automatic Feedback System}

In order to support presenters with an effective solution that can help them self-practice even at home, we aimed to implement the system with the following functions: (1)Automatic analyzes presenters performance; (2)Provides immediate feedback during the presentation; (3)Provides overall analysis about the whole presentation; (4)Lets users review their performance together with the analyzed results, thus allows them to keep track of their practicing progress. In order to achieve these purposes, we set up a Microsoft Kinect to extract body's skeletal representation as input for the analysis task. In parallel, a regular camera or webcam is positioned to simulate the audiences point of view. The automatic analysis, as well as recording is processed in real-time using a regular PC. The result is visualized on the PC or an external screen/projector (Figure 2). Users also have the chance to review their presentations, together with in-depth analysis about nonverbal cues in the end.

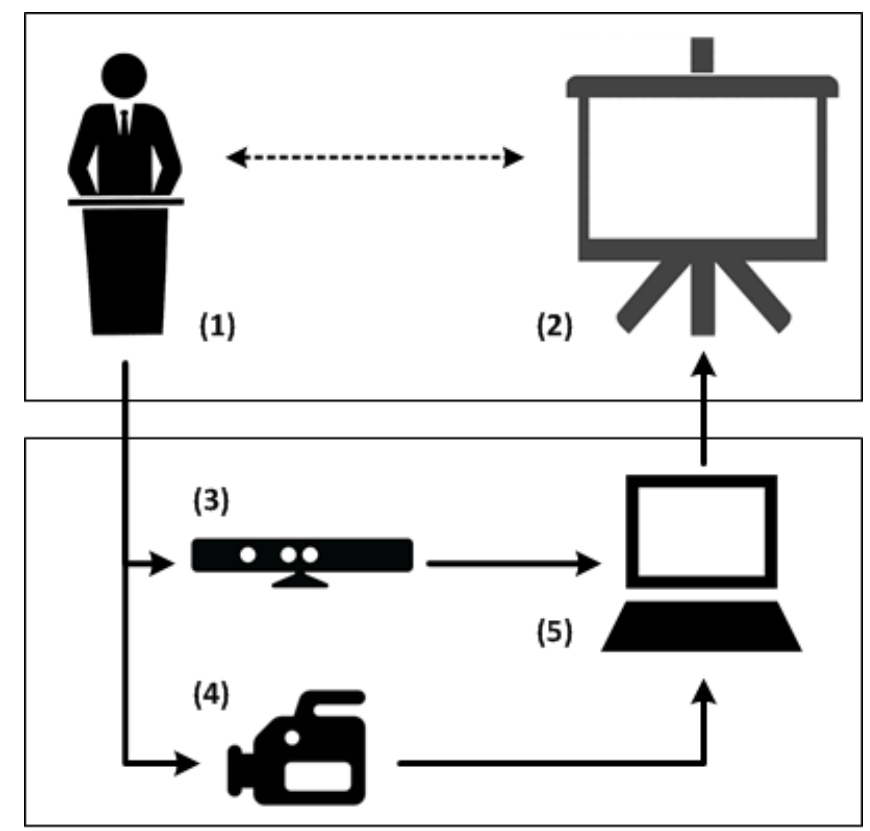

Fig. 2. Setup of the system 


\subsection{Recognition of Nonverbal Cues}

In order to implement a system that mostly takes visual nonverbal channels into account, together with conclusions from our observation, we currently focused on the four aspects: (1)Eye contact; (2)Posture; (3)Gesture; (4)Whole body movement. For each aspect, several related behaviors were recognized, using solely data captured from the Kinect device. They are listed as follows, readers can refer to Table 1 to link to the behavior associated to each number:

- Eye Contact: \#18, 19.

- Posture: \#2, 3, 4, 7, 8 .

- Gesture: \#28, 29.

- Movement: \#23, 24, 25, 26.

In the next subsections, we will explain the algorithms that were employed to detect these behaviors.

Eye Contact. Eye contact has significant impact on the creation and maintenance the connection between a presenter and the audience. Unfortunately, one common mistake was found in our observation is eye contact avoidance, which appeared in 28 over 39 presentations (Table 1, \#19). Our observation found that, losing eye contact with the audience is the quickest way to ruin a presentation. Thus, we aimed to recognize the moments that the visual attention of the presenters is shifted away from the audience.

Visual focus of attention has been widely employed for human-computer interaction and usability research [17]. However, these applications require several constrain, regarding the minimum resolution of input data, or users are required to wear specific devices. Furthermore, in public speaking, eye contact does not really means knowing exactly the eye movements. Instead, it more concerns whether the audience can perceive that they are being addressed. In many applications, instead of localizing gaze exactly, head/face orientation was used as the effective approximation for subjects focus target $[18,19]$. The experiment in [20] also proved that, head orientation was the reliable indication of the visual focus of attention in $89 \%$ of the time.

Our system relies on faces 3D orientation, which is provided as part of the Kinect SDK as the measurement for visual attention. In our observation, when not making eye contact, presenters mostly looked up to ceiling or down to floor. When these behaviors appear, the pitch angle of their faces drops to lower/raise to higher than some specific ranges. These ranges can be set manually based on the observation. In our implementation, we set the range of face's pitch angle that according to having good facial orientation is within $\left[-15^{0} ; 10^{0}\right]$. Being outside this range is assumed as eye contact avoidance appears.

Amount of Global Movement and Hand Gesture. Amount of movements and hand gesture can visually indicate the amount of energy that presenters use, also can reveal some hints about their internal states. Our observation results 
suggested that, this amount should be kept at the appropriate intensity to avoid negative impressions for the audience (Table 1, \#23, 24, 28, 29). The next issue is, how much movements/gesture is suitable? In order to answer this question, we extracted from the annotation data the durations that movements and hand gesture were annotated as too much/little. Next, the EyesWeb XMI [21] was used on the Kinect-recorded database to extract the skeletal movement in these durations. We used central position of upper body to measure the amount of whole body movement, and total distance of two hands for the amount of hand gesture. Finally, the average results were computed (Table 2).

Table 2. Average velocity of body movements and hand gestures when being too much or little (in meter/s)

\begin{tabular}{|l|c|c|c|}
\hline Behavior & $\mathrm{N}$ & Mean & $\mathrm{Sd}$ \\
\hline Too much movements & 42 & 0.34 & 0.08 \\
\hline Too little movements & 67 & 0.11 & 0.03 \\
\hline Too much hand gesture & 36 & 1.87 & 0.32 \\
\hline Too little hand gesture & 53 & 0.17 & 0.09 \\
\hline
\end{tabular}

We computed the average velocity of upper body and two hands in a moving time window of 5 seconds. Next, the mean values in Table 2 were used as hard thresholds to detect whether presenters' whole body and hands travelled too much/little.

Direction of Global Movement. Direction is one important characteristic of movement (Table 1, \#25, 26). Moving small steps forward brings presenters closer to the audience, thus expresses the willing to make connection. In contrast, small steps backward can be seen in awkward presenters, when they subconsciously retreat from the stage.

We aimed to detect these behaviors via analyzing the trajectories of the skeletal joint Spine, being projected to the ground floor. At each frame, we calculated the direction of displacement. This direction was compared with the orientation of the upper body that was determined via shoulders positions. The comparison output the decision whether the presenter shifted backward/forward in this frame. We accumulated the number of these frames in a moving window of 1 second. All of frames inside a moving window is regarded as positive if such window contains more than $80 \%$ positive frames.

Body Posture. Posture is the static configuration of the presenters' body. We focused on the two postural aspects, including foot position and the focus of body weight. Foot position relates to the behaviors \#2 and \#3 in Table 1. It can be easily measured via Euclidean distance of Foot_Left $\left(F_{C}\right)$ and Foot_Right $\left(F_{R}\right)$. This number was normalized by the distance of Shoulder_Left $\left(S_{L}\right)$ and Shoulder_Right $\left(S_{R}\right)$ to eliminate the effect of body size (Equation 1). 


$$
\text { Ratio }_{\text {FootDistance }}=\frac{\operatorname{distance}\left(F_{L}, F_{R}\right)}{\operatorname{distance}\left(S_{L}, S_{R}\right)}
$$

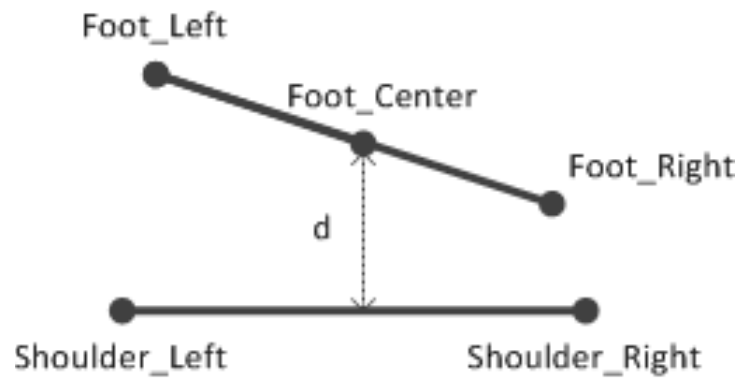

Fig. 3. Determine whether the presenter is leaning forward or backward via the distance $d$

Body weight relates to the behaviors \#7, 8 in Table 1. It was measured via the the positions of shoulders, in comparison with foot, being projected to the ground floor (Figure 3). The distance $d$ determines the degree of leaning, while the leaning backward or forward of body is determined via whether Foot_Center - $\left(F_{C}\right)$ is in the left or right side of the vector created by Shoulder_Left and Shoulder_Right.

Another behavior relates to body weight is whether a presenter is standing in one leg/feet. This degree of leaning is determined via comparing the distance between Hip_Center - $\left(H_{C}\right)$ with Foot_Left and Foot_Right (Equation 2).

$$
\text { Ratio }_{W \text { eight }}=\frac{\operatorname{distance}\left(H_{C}, F_{L}\right)-\operatorname{distance}\left(H_{C}, F_{R}\right)}{\operatorname{distance}\left(F_{L}, F_{R}\right)}
$$

Again, we relied on our Kinect-recorded database to find the thresholds for the above postural metrics. The method similar to the one used in Section 3.1 was used, this time for every single frames that the behaviors appeared. The process produced the results as shown in Table 3 .

Table 3. Average values for the postural metrics

\begin{tabular}{|l|c|c|c|}
\hline Postural metric & $\mathrm{N}$ & Mean & Sd \\
\hline Foot distance - closed (ratio) & 3982 & 0.48 & 0.18 \\
\hline Foot distance - stretch (ratio) & 1246 & 1.23 & 0.35 \\
\hline Weight forward (meters) & 2108 & 0.31 & 0.12 \\
\hline Weight backward (meters) & 2732 & 0.09 & 0.04 \\
\hline Weight on one feet (ratio) & 4120 & 0.88 & 0.19 \\
\hline
\end{tabular}


Table 4. Confusion matrix for the classification of the whole presentation

\begin{tabular}{|c|c|c|c|c|}
\hline \multirow{2}{*}{ Ground Truth } & \multicolumn{4}{|c|}{ Classified as } \\
\cline { 2 - 5 } & 1 & 2 & 3 & 4 \\
\hline 1 - Not good & $\mathbf{0 . 8 2 6}$ & 0.043 & 0.087 & 0.044 \\
\hline 2 - Good & 0.087 & $\mathbf{0 . 6 9 6}$ & 0.174 & 0.043 \\
\hline 3 - Very good & 0.087 & 0.130 & $\mathbf{0 . 6 5 2}$ & 0.131 \\
\hline 4 - Excellent & 0.043 & 0.043 & 0.043 & $\mathbf{0 . 7 8 3}$ \\
\hline & Average sensitivity: $\mathbf{0 . 7 3 9}$ \\
\hline
\end{tabular}

\section{Body Balance}

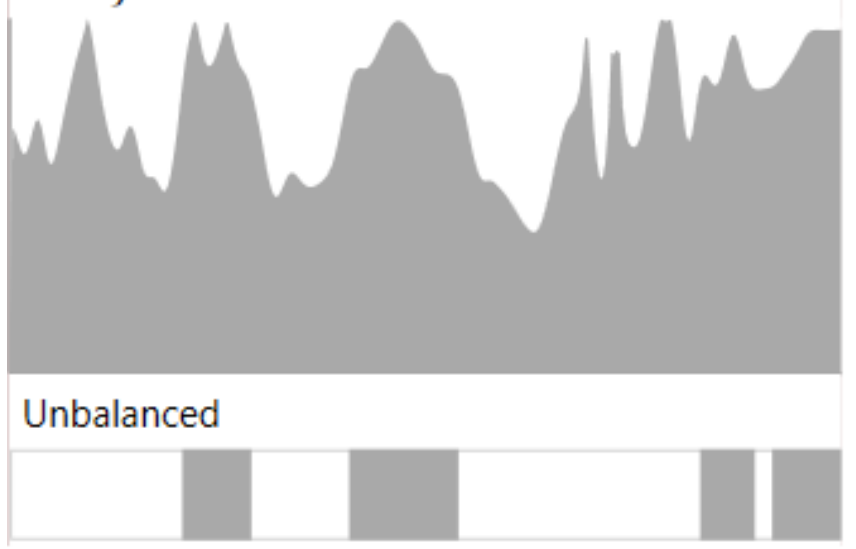

Fig. 4. Example of one behavior after being analyzed and displayed to presenters as off-line feedback. The upper graph visualizes the change of the metric used to measure body balance. The lower bar marks the durations that the behavior appeared.

Assessment of the Whole Presentation. After the separated behaviors can be recognized, we aimed to produce the final assessment for the whole presentation. Each presentation is assessed based on the four qualities, accordingly to the four nonverbal visual channels: (1) Posture; (2) Gesture; (3) Global Movement; (4) Eye contact. Additionally, one single quality is measured accordingly to the general performance. This is similar to what learners are given in most training classes about public speaking skills.

Firstly, in order to enrich the database, we asked for the permission to copy the DVDs of presentations from other students who attended the training class (each student is given one DVD that contains all of their presentations during the course in the last session). These video clips are not appear previously in our recorded database. Finally, we achieved extra 37 presentations (without Kinect recordings), in addition to our existing 39 videos that were used for the observation (which contain Kinect recordings). The expert was asked to assess the presentations based on a 4-level scale for each single aspect: (1) Posture; (2) 


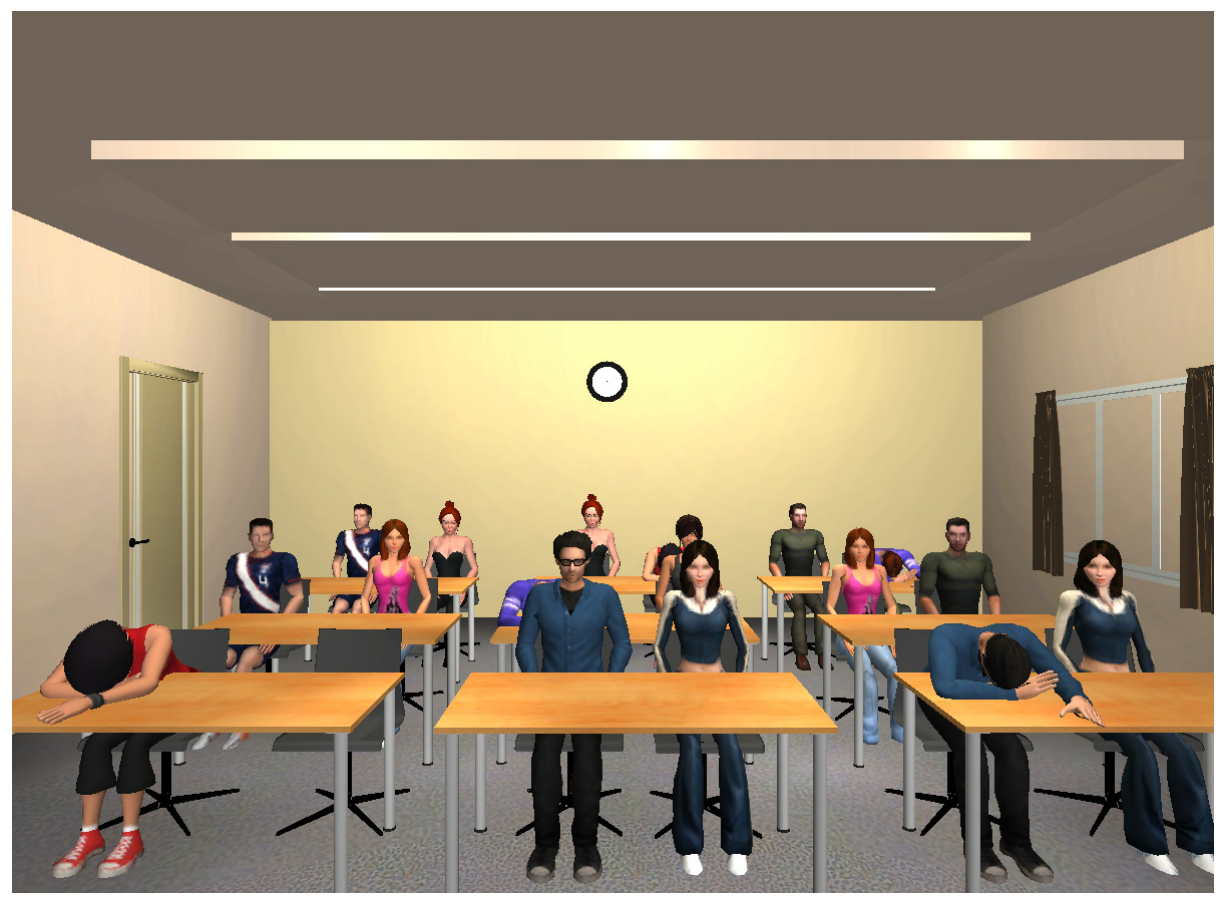

Fig. 5. The simulated conference room as the method to deliver immediate feedbacks. The avatars can perform different animations based on the performance of presenters.

Gesture; (3) Global Movement; (4) Eye contact, plus one score for the overall assessment. This data will be used for the later classification.

Based on the previous recognition process, a feature vector is created for each aspect, in which each component stands for the percentage that one single behavior appeared in the whole presentation. For example, feature vectors that represent hand gesture contain two dimensions for gesturing too much/little. Similarly, the number of dimensions of the feature vectors of movement, posture and eye contact are: 4, 5 and 1, respectively. Additionally, the whole presentations are represented via 12-component vectors, is the serialization of all features.

Amongst them, eye contact is represented via one single number and can be easily classified using hard thresholds. For the rest, feature vectors were used as the input for a multi-class Support Vector Machine system [11], using one-versusall method and winner-take-all strategy. The algorithm was used to classify four classes, which represented the performance from [1 - Not good] to [4 - Excellent].

We applied 70-30\% train/test split on the total of 76 presentations in order to evaluate the classification of the whole presentation, and then calculate the confusion matrix (Table 4). As the result, the system achieved the recognition rate of $73.9 \%$. 
The Two Methods of Giving Feedbacks. The system provides two ways of delivering feedbacks to the audience. The first one shows users their recorded presentation, the appearances of each behaviors (Figure 4) and results on the four nonverbal aspects, plus the overall result. In parallel, with purpose to give presenters the helpful feedbacks, also aim to provide them the experience as presenting for the real audience, we developed a virtual conference room as one method to deliver feedbacks. The environment was built using the Unity3D engine, simulates the classroom that we collected data for observation. Avatars are able to perform several animations that may bring either positive or negative feeling for presenters. These animation clips are sorted based on the increase of negative feeling: (1) Nodding; (2) Sitting still; (3) Sleeping; (4) Yawning.

In order to achieve real-time assessment, we applied a moving window to assess the overall quality for the most recent 5 seconds. This process outputted a single value from [ 1 - Not good] to [4 - Excellent]. The value then was used to manually adjust the distribution of the animation clips. Higher quality presentations resulted in higher proportion of positive animations and vice versa.

Acknowledgments. This work was supported in part by the Erasmus Mundus Joint Doctorate in Interactive and Cognitive Environments (ICE), which is funded by the Edu- cation, Audiovisual and Culture Executive Agency of the European Commission under EMJD ICE FPA 2010-0012.

\section{References}

1. Seiler, W.J., Beall, M.L.: Communication - Making connections. Allyn\&Bacon (2004)

2. Rodman, G., Adler, R.B.: Style: delivery and language choices. In: The New Public Speaker, 1st edn. Wadsworth Publishing (1996)

3. Argyle, M., Alkema, F., Gilmour, R.: The communication of friendly and hostile attitudes by verbal and nonverbal signals. European Journal of Social Psychology 1, 385-402 (1971)

4. D'Arcy, J.: Communicating with effective body language. In: Technically Speaking. Battelle Press, ch. 14 (1998)

5. Vinciarelli, A., Pantic, M., Bourlard, H.: Social signal processing: Survey of an emerging domain. Image and Vision Computing 27(12), 1743-1759 (2009)

6. Picard, R.: Affective Computing, 1st edn. The MIT Press (2000)

7. Hincks, R., Edlund, J.: Promoting increased pitch variation in oral presentations with transient visual feedback. Language Learning \& Technology 13(3), 32-50 (2009)

8. Kurihara, K., Goto, M., Ogata, J.: Presentation sensei: a presentation training system using speech and image processing. In: Proceedings of the 9th International Conference on Multimodal interfaces, pp. 358-365 (2007)

9. Pfister, T., Robinson, P.: Real-Time Recognition of Affective States from Nonverbal Features of Speech and Its Application for Public Speaking Skill Analysis. IEEE Transactions on the Affective Computing, 1-14 (2011). http://ieeexplore.iee.org/ xpls/abs_all.jsp?arnumber $=5740838$ 
10. Silverstein, D.A., Tong, Z., Zhang, T.: System and method of providing evaluation feedback to a speaker while giving a real-time oral presentation. US Patent 7,050,978 (2003)

11. Duan, K.-B., Keerthi, S.S.: Which is the best multiclass SVM method? An empirical study. In: Oza, N.C., Polikar, R., Kittler, J., Roli, F. (eds.) MCS 2005. LNCS, vol. 3541, pp. 278-285. Springer, Heidelberg (2005). http://link.springer.com/chapter/10.1007/11494683_28

12. Gao, T., Wu, C., Aghajan, H.: User-centric speaker report: Ranking-based effectiveness evaluation and feedback. In: 2009 IEEE 12th International Conference on Computer Vision Workshops (ICCV Workshops), pp. 1004-1011. IEEE (2009)

13. Freund, Y., Iyer, R., Schapire, R., Singer, Y.: An efficient boosting algorithm for combining preferences. The Journal of Machine Learning Research 4, 933-969 (2003). http://dl.acm.org/citation.cfm?id=964285

14. Nguyen, A., Chen, W., Rauterberg, G.: Feedback system for presenters detects nonverbal expressions. In: SPIE Newsroom (2013). http://spie.org/x91885.xml? highlight $=\mathrm{x} 2410 \&$ ArticleID $=\mathrm{x} 91885$

15. Zimmerman, P., Bolhuis, J.: The Observer XT: A tool for the integration and synchronization of multimodal signals. Behavior Research Methods 41(3), 731735 (2009). http://link.springer.com/article/10.3758/BRM.41.3.731

16. Kleinke, C.L.: Gaze and eye contact: a research review. Psychological Bulletin 100(1), 78-100 (1986)

17. Jacob, R., Karn, K.: Eye tracking in human-computer interaction and usability research: Ready to deliver the promises. Work 2(3), 573-605 (2003). http://www. ee.uwa.edu.au/ roberto/research/projects2013/10.1.1.100.445.pdf

18. Sheikhi, S., Odobez, J.-M.: Recognizing the visual focus of attention for human robot interaction. In: Salah, A.A., Ruiz-del-Solar, J., Meriçli, Ç., Oudeyer, P.-Y. (eds.) HBU 2012. LNCS, vol. 7559, pp. 99-112. Springer, Heidelberg (2012). http://link.springer.com/chapter/10.1007/978-3-642-34014-7_9

19. Ba, S.O., Odobez, J.-M.: Recognizing visual focus of attention from head pose in natural meetings. IEEE transactions on systems, man, and cybernetics. Part B, Cybernetics : a publication of the IEEE Systems, Man, and Cybernetics Society 39(1), 16-33 (2009). http://www.ncbi.nlm.nih.gov/pubmed/19068430

20. Stiefelhagen, R.: Tracking focus of attention in meetings. In: Proceedings of the Fourth IEEE International Conference on Multimodal Interfaces, pp. 273-280. IEEE Comput. Soc (2002). http://ieeexplore.iee.org/lpdocs/epic03/wrapper. htm?arnumber $=1167006$

21. Camurri, A., Hashimoto, S., Ricchetti, M., Ricci, A., Suzuki, K., Trocca, R., Volpe, G.: Eyesweb: Toward gesture and affect recognition in interactive dance and music systems. Computer Music Journal 24(1), 57-69 (2000) 